\title{
Uso de geotecnologia na pesquisa bibliográfica: Biblioteca geoespacial sobre os recursos hídricos de Sergipe
}

Use of geotechnology in bibliographic research: Geospatial library on the water resources of Sergipe

\author{
J. C. S. da Rocha*; C. H. Santos ; S. L. Rocha \\ Superintendência de Recursos Hídricos, Secretaria de Estado do Meio Ambiente e dos Recursos Hídricos, 49030-640, \\ Aracaju-SE, Brasil
}

*joaocarlos.rocha@semarh.se.gov.br

(Recebido em 19 de junho de 2017; aceito em 28 de setembro de 2017)

\begin{abstract}
As diversas esferas de governo despendem anualmente uma significativa soma de recursos financeiros na realização de estudos sobre diversos temas que, na grande maioria das vezes, ficam esquecidos e longe do alcance da sociedade e dos meios acadêmicos e científicos. O desconhecimento dessas pesquisas ocasiona o retrabalho e a falta de continuidade no seu avanço em busca de um aprimoramento sobre o tema pesquisado. Esse trabalho objetivou apresentar uma alternativa para disponibilização de estudos realizados na área de meio ambiente e dos recursos hídricos, através de uma pesquisa bibliográfica utilizando ferramentas de geotecnologia. O projeto, desenvolvido pela Secretaria de Estado do Meio Ambiente e dos Recursos Hídricos (SEMARH), denominado Biblioteca Geoespacial sobre os Recursos Hídricos de Sergipe, espacializou a área de abrangência de 947 estudos realizados em Sergipe e agrupados em 14 temas. A metodologia desenvolvida e os resultados iniciais indicam um grande potencial da ferramenta, que poderá se tornar um depositório para trabalhos técnico-científicos e projetos, com consulta espacial.
\end{abstract}

Palavras-chave: bibliografia, QGis, software livre

The various spheres of government spend annually a significant sum of financial resources in conducting studies on a variety of topics that are, for the most part, forgotten and out of reach of society and academic and scientific circles. The lack of knowledge of these research results in the reworking and lack of continuity in its progress in search of an improvement on the researched topic. This work aimed to present an alternative for the availability of studies in the area of environment and water resources, through a bibliographical research using geotechnology tools. The project, developed by the State Secretariat for the Environment and Water Resources (SEMARH), called the Geospatial Library on Water Resources in Sergipe, spatialized the coverage area of 947 studies carried out in Sergipe and grouped into 14 topics. The methodology developed and the initial results indicate a great potential of the tool, which could become a depository for technicalscientific works and projects, with spatial query.

Keywords: bibliography, QGis, open source software

\section{INTRODUÇÃO}

Desde o ano de 2000, os investimentos em Ciência, Tecnologia e Inovação (CT\&I) do Governo Federal têm sido elevados a taxas superiores ao crescimento econômico. No ano de 2013, os dispêndios em CT\&I pelo governo federal e pelos estados alcançaram o valor de R \$ 47,9 bilhões [1]. Apenas no âmbito da Superintendência de Recursos Hídricos de Sergipe, nos últimos 20 anos, foram investidos cerca de $\mathrm{R} \$ 20$ milhões em estudos e projetos na gestão das águas.

Esse montante de recursos financeiros aplicados gerou informações, que, se adequadamente sistematizadas e disponibilizadas, podem criar um ambiente incremental e sinérgico para futuros estudos.

No entanto, geralmente, o que acontece é que todo esse conhecimento produzido acaba esquecido em prateleiras, dispersos em vários sites de internet e na memória dos técnicos e pesquisadores que desenvolveram ou acompanharam os estudos e projetos.

Assim, procurando minimizar essa situação e utilizando a experiência adquirida em geotecnologia com o desenvolvimento, desde 2004, do Atlas Digital sobre os Recursos Hídricos de Sergipe [2], ganhador do Prêmio ANA 2012 na categoria Governo, a Secretaria de Estado do 
Meio Ambiente e dos Recursos Hídricos (SEMARH) lançou em 2016 a Biblioteca Geoespacial sobre os Recursos Hídricos de Sergipe.

O projeto catalogou, com auxílio de estudantes da Universidade Federal de Sergipe (UFS) e técnicos da SEMARH, centenas de estudos que foram agrupados em temas afins. A disponibilização dos dados dá-se em um ambiente de Sistemas de Informações Geográficas (SIG), por meio de um programa open source (QGIS), onde o usuário busca os estudos disponíveis e catalogados selecionando no mapa a região de seu interesse.

Para facilidade do usuário, tem-se como cenário de fundo a divisão política do Estado e as bacias/sub-bacias hidrográficas, sendo possível incorporar ainda todas as camadas (arquivos shapefile) disponibilizadas pelo atlas digital.

O uso do SIG, como ferramenta auxiliar para a pesquisa bibliográfica, fundamentou-se no fato de que quase todas as instituições que desenvolvem ou utilizam ciência, tecnologia e inovação, sejam universidades, institutos de pesquisas e empresas, utilizam essa ferramenta de geotecnologia. Além do mais, como pontuam Bolfe et al. (2008) [3], o domínio da informação espacial permite analisar fatores do passado e presente, permitindo antever futuras transformações do espaço geográfico, especialmente, se considerarmos a possibilidade do cruzamento de vários tipos de dados, informações e estudos empreendidos.

Assim, o presente trabalho tem como objetivo apresentar uma proposta inovadora para armazenar, organizar e localizar estudos relacionados ao meio ambiente e aos recursos hídricos, utilizando um software livre de geotecnologia (QGis), através de uma consulta espacial e tendo a possibilidade de cruzar diversas camadas (layers) relacionadas ao meio físico, social, político e econômico com os estudos e projetos realizados numa dada região.

\section{MATERIAL E MÉTODOS}

A Biblioteca Geoespacial sobre os Recursos Hídricos foi construída com base no levantamento dos estudos e projetos realizados no Estado, relacionados ao meio ambiente e aos recursos hídricos, e apoiada na cartografia digital consolidada pelo Atlas Digital sobre Recursos Hídricos de Sergipe, versão 2016.10.

Os estudos e projetos foram catalogados por Battesini et al. (2013) [4], que levantou 272 estudos existentes nas unidades de conservação de Sergipe, e Ferrão (2015) [5], tendo cadastrado 526 trabalhos relativos aos recursos hídricos.

Os referidos autores cadastraram a bibliografia por meio de buscas eletrônicas nos seguintes sites: CAPES, Google Acadêmico e Scielo, além do banco de dados dos cursos de pós-graduação da UFS. Os nomes dos pesquisadores levantados na primeira etapa foram então analisados, através de seus currículos, na plataforma Lattes, em busca por mais trabalhos.

Os estudos foram então agrupados por temas e sistematizados em planilhas eletrônicas do Excel, onde os trabalhos duplicados foram eliminados.

$\mathrm{O}$ acervo produzido foi atualizado e complementado pelos técnicos da Superintendência de Recursos Hídricos (SRH), que incluíram outros sites de pesquisa, com destaque para repositório Acesso Livre à Informação Científica da Embrapa (Alice).

Os campos levantados, que se tornaram futuros atributos nas tabelas do programa QGIS, foram: assunto/tema, título, autor, ano, tipo, formato, onde encontrar, palavras-chave, abrangência e link para acesso.

Os trabalhos científicos foram agrupados nos 14 temas/assuntos listados a seguir: Agricultura/Irrigação, Climatologia/Meteorologia, Dinâmica Ambiental/Alteração da Paisagem, Educação Ambiental, Fauna, Flora, Gestão e Planejamento, Hidrologia/Hidrogeologia, Impactos Ambientais, Meio Físico, Qualidade da Água, Química Ambiental, Reflorestamento/Recuperação de Áreas e Relações Sócio-ambientais/Conflitos.

Cada um dos trabalhos resultantes do acervo final teve sua área de abrangência delimitada no programa QGIS, versão de longo prazo 2.14.3-2.

Estudos que possuíam áreas semelhantes e pertencentes a um mesmo tema foram unidos nas ferramentas de geoprocessamento do software open source. 
Visando diminuir o tamanho dos arquivos shapefile resultantes, foi realizada uma simplificação da geometria das áreas de abrangência, utilizando uma tolerância compatível para cada arquivo. Após esse último procedimento, a velocidade para a pesquisa bibliográfica utilizando a ferramenta QGIS foi incrementada e mostrou-se adequada para a maioria dos processadores de dados.

Por fim, a transparência foi aplicada a todas as linhas e preenchimentos das áreas de abrangência com o fito de destacar apenas o mapa base, onde o usuário irá localizar a área de seu interesse.

O mapa base incluído na Biblioteca Geoespacial sobre os Recursos Hídricos foi retirado do Atlas Digital sobre Recursos Hídricos de Sergipe, versão 2016.10, de onde foram incorporadas as seguintes camadas para servirem de orientação ao usuário para a seleção da área de interesse: hidrografia (lagos e reservatórios, rios principais, estuários e rio São Francisco), divisão hidrográfica (bacia hidrográfica e unidades de planejamento), transporte (rodovia estadual e federal), divisão política (localidades, sedes municipais, limites municipais, estados do Brasil e oceano) e o modelo digital de elevação.

Objetivando ampliar o universo de usuários, foi implementada uma interface amigável que permite, através de botões de fácil acesso, instalar o programa e abrir o projeto QGIS, apresentar um tutorial e abrir um canal para o envio de comentários e de complementações aos estudos atualmente incluídos na Biblioteca.

A Figura 1 apresenta, detalhadamente, as atividades desenvolvidas na elaboração da Biblioteca Geoespacial sobre os Recursos Hídricos.

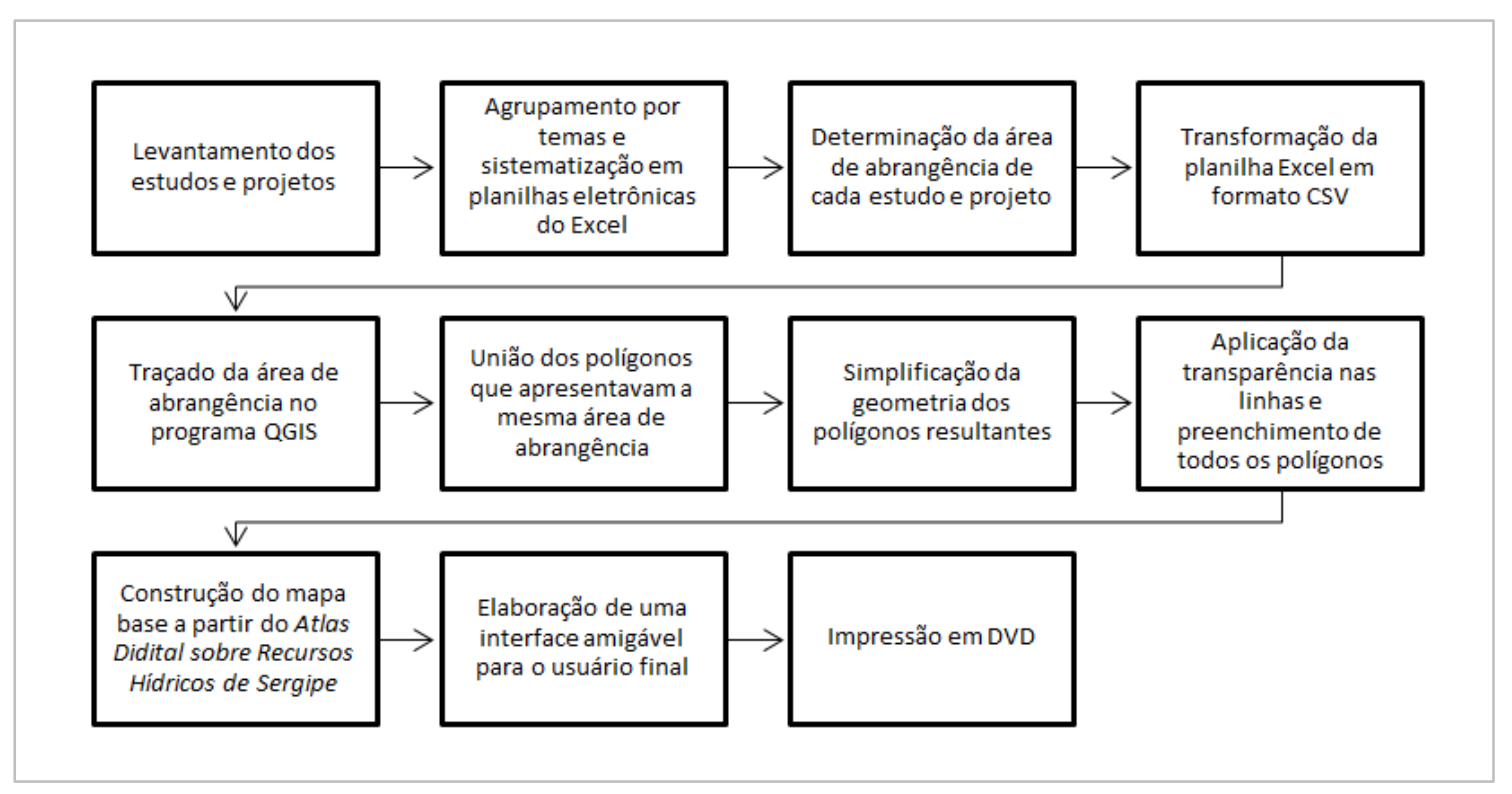

Figura 1: Tela da Biblioteca Geoespacial sobre os Recursos Hídricos.

\section{RESULTADOS E DISCUSSÃO}

O levantamento dos estudos e projetos relativos ao meio ambiente e recursos hídricos de Sergipe resultou num total de 947 trabalhos, o qual foi realizado até novembro de 2016.

Vale a pena salientar que este número representa ainda uma pequena fração dos trabalhos que foram realizados ao longo dos anos em Sergipe. Especialmente nas diferentes esferas de governo, diversas instituições possuem muitos trabalhos que se encontram apartados das mídias de ampla difusão de informações e, portanto, não puderam ser acrescentados nesta versão.

A Tabela 1 mostra o número e o percentual de trabalhos disponibilizados na Biblioteca Geoespacial sobre os Recursos Hídricos.

Observa-se que o assunto Gestão e Planejamento representa o maior número de estudos levantados, seguido pelo tema Flora, especialmente pela grande quantidade de pesquisas realizadas no Parque Nacional da Serra de Itabaiana. 
Tabela 1: Número e percentual de trabalhos cadastrados por tema

\begin{tabular}{lcc}
\hline \multicolumn{1}{c}{ Tema } & $\begin{array}{c}\text { Número de } \\
\text { Trabalhos }\end{array}$ & \% \\
\hline Agricultura e Irrigação & 51 & 5,4 \\
\hline Climatologia e Meteorologia & 36 & 3,8 \\
\hline Dinâmica Ambiental e Alteração da Paisagem & 94 & 9,9 \\
\hline Educação Ambiental & 60 & 6,3 \\
\hline Fauna & 87 & 9,2 \\
\hline Flora & 117 & 12,4 \\
\hline Gestão e Planejamento & 171 & 18,1 \\
\hline Hidrologia e Hidrogeologia & 40 & 4,2 \\
\hline Impactos Ambientais & 76 & 8,0 \\
\hline Meio Físico & 36 & 3,8 \\
\hline Qualidade da Água & 54 & 5,7 \\
\hline Química Ambiental & 27 & 2,9 \\
\hline Reflorestamento e Recuperação de Áreas & 24 & 2,5 \\
\hline Relações Socioambientais e Conflitos & 74 & 7,8 \\
\hline TOTAL & 947 & 100,0 \\
\hline
\end{tabular}

Como mencionado anteriormente, foi elaborada uma interface amigável ao usuário final, que com um conhecimento básico de informática, pode acessar o projeto da Biblioteca já no programa QGIS. Foi utilizado para tal finalidade o programa AutoRun Pro, versão 8.0.5.140.

A Figura 2 apresenta a tela inicial que deverá ser carregada automaticamente com a inserção do DVD no computador.

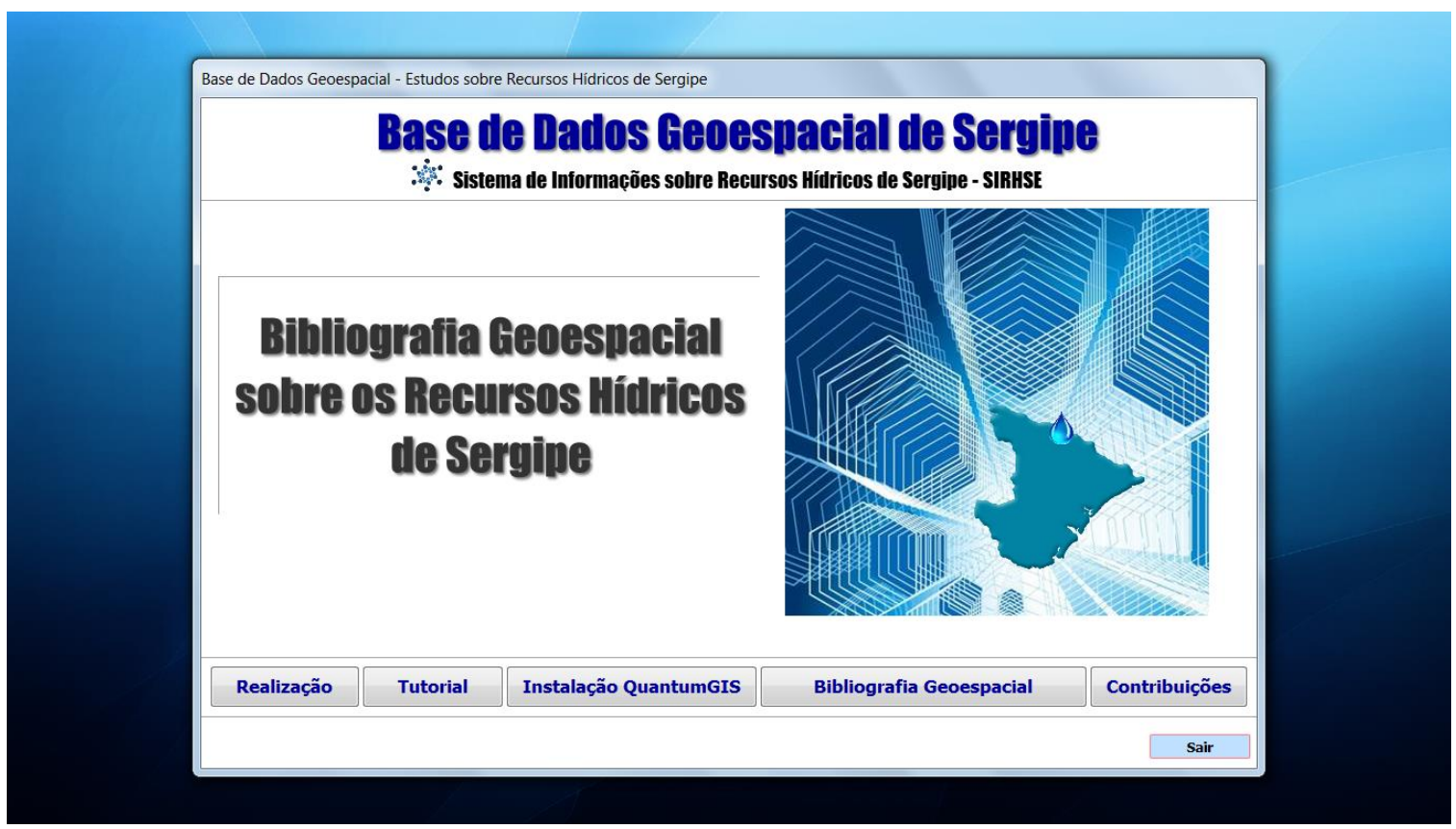

Figura 2: Tela da Biblioteca Geoespacial sobre os Recursos Hídricos.

A pesquisa bibliográfica realizada pelo usuário final na Biblioteca Geoespacial sobre os Recursos Hídricos, utilizando o QGIS, pode ser executada de forma simples e rápida.

Os seguintes passos devem ser executados para sua utilização:

1) Instalação do programa QGIS, esse procedimento deverá ser executado apenas uma vez, e poderá ser feito através de botão apropriado na tela principal, conforme apresentada na Figura 2;

2) Em seguida, o projeto QGIS (formato .qgs) da Biblioteca Geoespacial poderá ser aberto acionando o botão correspondente;

3) Para dar início à pesquisa, deve-se selecionar o tema de interesse no painel "camadas"; 
4) Após, com a ferramenta "identificar feições" acionada, procede-se a seleção da área de interesse, tendo como orientação o mapa base de preferência (municípios, bacias hidrográficas etc.). A ferramenta zoom pode ser empregada para propiciar um maior detalhamento, bem como, incluir outras camadas, tais como estradas e localidades.

A Figura 3 exibe como exemplo a tela com o resultado para uma pesquisa bibliográfica na região de Canindé de São Francisco, próximo ao Projeto Irrigado Califórnia. Observe a tabela apresentada pelo QGIS com o atributo "Título" de todos os trabalhos realizados que tiveram alguma relação com o ponto selecionado no mapa.

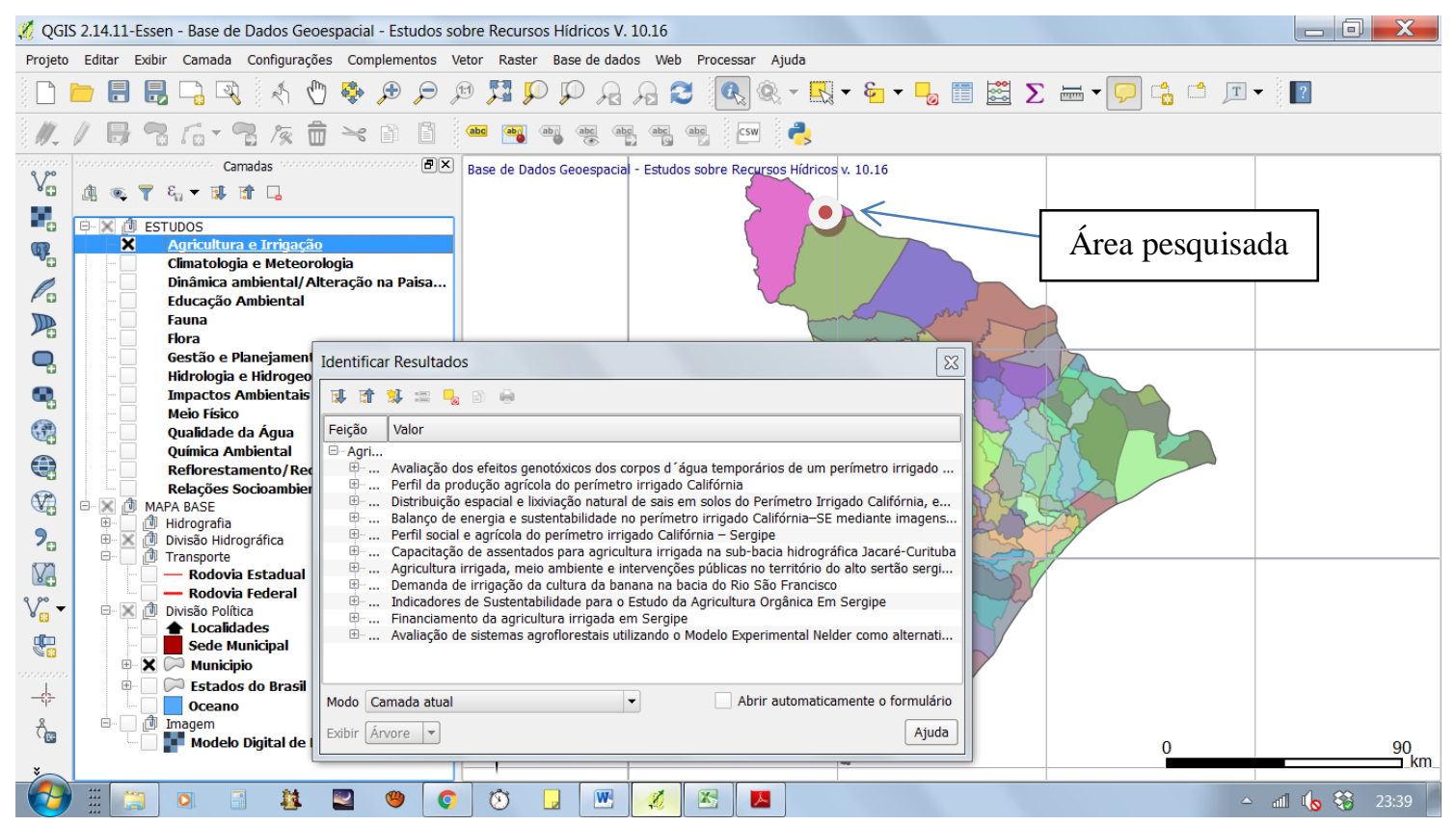

Figura 3: Tela da Biblioteca Geoespacial sobre os Recursos Hídricos.

A utilização de uma ferramenta já consolidada no meio técnico, como é o caso de Sistemas de Informações Geográficas - SIG, de forma não usual, como a pesquisa bibliográfica espacializada, requer certo esforço por parte dos usuários em direção a essa nova aplicação.

Em Sergipe, observa-se que, apesar da ampla distribuição por parte da SEMARH da Biblioteca Geoespacial sobre os Recursos Hídricos, as instituições governamentais e o meio técnico-científico ainda não se atentaram para todo o potencial que essa nova forma de utilizar o SIG pode acrescentar ao conhecimento.

\section{CONCLUSÃO}

A pesquisa bibliográfica através de mapas, como apresentada neste artigo, é uma forma inovadora de consulta e armazenamento de documentos técnico-científicos.

A Biblioteca Geoespacial sobre os Recursos Hídricos pode representar uma importante ferramenta como forma de disponibilizar informações sobre recursos hídricos e temas afins (meio ambiente, projetos de infraestrutura hídrica, planejamento territorial, dentre outros). No entanto, torna-se imprescindível a apropriação, por parte das instituições técnico-científicas e órgãos governamentais, desta ferramenta com o intuito de efetuar atualizações periódicas.

Isso possibilitará uma razoável economia de recursos financeiros e esforços técnicos, que poderão ser direcionados à atualização dos estudos existentes e à elaboração de pesquisas complementares.

No entanto, apesar de ter sido utilizada nesse projeto uma plataforma SIG gratuita, objetivando alcançar um maior número de usuários, a distribuição da Biblioteca Geoespacial através de mídia ótica (DVD) ainda constitui uma restrição ao grande público. Assim, como pontuam De Carvalho e Di Maio (2011) [6], a informação geoespacial disponibilizada gratuitamente pela Internet 
constitui um estímulo à adoção de novas tecnologias inseridas no âmbito da cibercultura que atualmente vivenciamos. O que deverá ser buscada pela SEMARH para que seus objetivos sejam plenamente alcançados.

\section{AGRADECIMENTOS}

Os autores agradecem à Universidade Federal de Sergipe (UFS), através do Departamento de Ciências Florestais, pela iniciativa de incluir nas linhas de pesquisa que direcionam os Trabalhos de Conclusão de Curso (TCC) temas afins aos projetos da SEMARH.

\section{REFERÊNCIAS BIBLIOGRÁFICAS}

1. BRASIL. Ministério da Ciência, Tecnologia e Inovação. Estratégia Nacional de Ciência, Tecnologia e Inovação - 2016-2019. Brasília, DF; 2016. 128p. Disponível em: 05/05/2017

2. SERGIPE. Atlas Digital sobre Recursos Hídricos de Sergipe. Aracaju, SE: Secretaria de Estado do Meio Ambiente e dos Recursos Hídricos, 2016. CD-ROM.

3. Bolfe EL, Matias LF, Ferreira MC. Sistemas de Informação Geográfica: Uma abordagem contextualizada na história. Geografia. 2008;33(1):69-88.

4. Battesini MD, Santos SZA dos, Melo Neto J de O, Gomes LJG. Publicações acadêmicas das unidades de conservação no estado de Sergipe, Brasil. Interciência. 2013;38(1):67-72.

5. Ferrão CS. Coletânea Bibliográfica Acadêmica sobre os Recursos Hídricos de Sergipe. [monografia]. São Cristóvão (SE): Universidade Federal de Sergipe; 2015. 122 p.

6. De Carvalho MVA, Di Maio AC. Proposta para a difusão de dados e informações geoespaciais disponíveis gratuitamente junto aos graduandos e professores da educação básica. In: Anais do XV Simpósio Brasileiro de Sensoriamento Remoto; 2011. Curitiba: INPE. p. 3351-3358. 